\title{
Lapatinib sensitizes quiescent MDA-MB-231 breast cancer cells to doxorubicin by inhibiting the expression of multidrug resistance-associated protein-1
}

\author{
YUN-SUK KWON, SO-YOUNG CHUN, KYUNG-SOO NAM and SOYOUNG KIM \\ Department of Pharmacology, School of Medicine and Intractable Disease Research Center, \\ Dongguk University, Gyeongsangbuk-do 780-350, Republic of Korea
}

Received March 13, 2015; Accepted April 27, 2015

DOI: $10.3892 /$ or.2015.4047

\begin{abstract}
The quiescent state plays an important role in tumor recurrence because it protects cancer cells from chemotherapy. Previously, we optimized tumorsphere cultures for in vitro screening methods for targeting quiescent cell population since the majority of cells in tumorspheres are quiescent. In this study, we analyzed efficacies of current chemotherapeutics in tumorsphere assays to seek better strategies for eradicating quiescent cell population. Tumorspheres generated from MDA-MB-231 cells exhibited accumulations of cells in the G0/G1 phase as compared with cells in monolayer culture, suggesting that sphere formation contributes to an increase of quiescent cells. As a result of a decreased doxorubicin uptake, MDA-MB-231 tumorspheres exhibited chemoresistance to both doxorubicin and paclitaxel. Since we found that the enhanced EGFR signaling is characteristics of MDA-MB231 tumorspheres, the combination effects of chemotherapy with lapatinib, a dual ErbB1/ErbB2 inhibitor, were accessed in tumorsphere assays. Western blot analysis revealed that lapatinib inhibited the phosphorylation of EGFR, AKT and
\end{abstract}

Correspondence to: Dr Soyoung Kim or Dr Kyung-Soo Nam, Department of Pharmacology, School of Medicine and Intractable Disease Research Center, Dongguk University, 87 Dongdae-ro, Gyeongju-si, Gyeongsangbuk-do 780-350, Republic of Korea E-mail: soyoungkim@dongguk.ac.kr

E-mail:namks@dongguk.ac.kr

Abbreviations: TS, tumorsphere; 2D, two dimensional; Lap, lapatinib; Dox, doxorubicin; ABC, ATP-binding cassette; MRP-1, multidrug resistance-associated protein-1; MDR-1, multidrug resistance protein-1; BCRP, breast cancer resistance protein; HER2, human epidermal growth factor receptor 2; ER, estrogen receptor; PR, progesterone receptor; EGFR, epidermal growth factor receptor; PI3K, phosphoinositol-3 kinase; MAPK, mitogen-activated protein kinase; AKT, protein kinase B; ERK, extracellular signal-regulated kinase; GAPDH, glyceraldehyde-3-phosphate dehydrogenase

Key words: tumorsphere, quiescence, chemoresistance, lapatinib, doxorubicin, MDA-MB-231 breast cancer cells, MRP-1 p38 in doxorubicin-treated tumorspheres. The inhibition of EGFR signaling by the treatment with lapatinib suppressed the expression of multidrug resistance-associated protein-1 (MRP-1), leading to increased cytotoxicity of doxorubicin to tumorspheres. Furthermore, blockade of the PI3K/AKT and p38 MAPK signaling pathways resulted in a remarkable decrease in the expression of MRP-1 in doxorubicin-treated tumorspheres. These results demonstrate that lapatinib sensitizes quiescent MDA-MB-231 breast cancer cells to doxorubicin by inhibiting doxorubicin-induced MRP-1 expression via PI3K/AKT and p38 MAPK signaling pathways. Thus, this study suggests that treatment with lapatinib in combination with anti-mitotic drugs maybe a useful approach to improve clinical response by eradicating the quiescent cancer cell population.

\section{Introduction}

Chemoresistance is a major cause of cancer treatment failure. The mechanisms involved in the development of chemoresistance are complex and not fully understood. Since conventional chemotherapies target proliferating cells and require active cycling for induction of apoptosis, it has been proposed that cells in the quiescent state within tumors are associated with resistance and cell survival in chemotherapies (1). Moreover, the quiescent nature of cells is known to be characteristic of cancer stem cells, which have the ability to self-renew and differentiate to produce heterogeneous tumor cell lineages (1-3). Increasing evidence indicates that cancer stem cells are responsible for treatment failure and tumor recurrence because their quiescent nature is likely to contribute to the survival in response to chemotherapy (4). Thus, selectively targeting quiescent cell population including cancer stem cells offers possible way forward to overcome chemoresistance and improve the clinical outcomes of cancer patients.

The sphere culture has been proposed to propagate cells with stem cell properties and has been widely adopted to study stem cell biology (5-8). It is a relatively easy, rapid and non-animal-depending model to assess stem cell activity, but the application of sphere-forming assays for high-throughput screening is limited due to formation of variable sizes of spheres and lack of a simple and well-established analytical 
tool. Moreover, cell aggregation in spheres can cause misinterpretation. Previously, we showed that sphere cultures exhibit higher proportions of quiescent cells and we optimized tumorsphere cultures for the in vitro screening of chemotherapeutics against the quiescent cell population (9).

In this study, we utilized tumorsphere cultures to identify better ways of eradicating quiescent tumor cell population in MDA-MB-231 human breast cancer cells. MDA-MB-231 cells are representative of triple-negative breast tumors, which are characterized by the absence of estrogen receptor (ER), progesterone receptor (PR) and human epidermal growth factor receptor 2 (HER2) (10). This triple negative tumor subtype is mainly correlated to poor outcomes, showing the worst overall and disease-free survival rates due to a lack of effective targeted therapies $(11,12)$. Thus, cytotoxic chemotherapies such as doxorubicin/paclitaxel remain the mainstay of treatment for triple-negative breast cancer, but resistance is common and can develop rapidly (13). To seek better strategies to overcome chemoresistance, we analyzed the efficacies of chemotherapeutics, doxorubicin and paclitaxel, on MDA-MB231 tumorspheres. Since we found that the enhanced epidermal growth factor receptor (EGFR) signaling pathway is characteristic of MDA-MB-231 tumorspheres, we assessed the combination effects of doxorubicin and lapatinib (a dual ErbB1/ ErbB2 inhibitor) in tumorsphere assays.

\section{Materials and methods}

Adherent cell culture. The MDA-MB-231 human breast cancer cell line was purchased from the Korean Cell Line Bank (Seoul, Korea) and routinely maintained in DMEM (Welgene, Daegu, Korea) supplemented with $10 \%$ fetal bovine serum (Invitrogen, Carlsbad, CA, USA) and 1\% antibioticantimycotic solution (Welgene).

Suspension sphere cultures. The protocol used for tumorsphere culture was as previously described $(5,6,8,14)$. Briefly, cells were suspended in serum-free DMEM/F12 (Welgene) supplemented with 1:50 B27 (Gibco BRL, Grand Island, NY, USA), $10 \mu \mathrm{g} / \mathrm{ml}$ insulin (Welgene), $20 \mathrm{ng} / \mathrm{ml} \mathrm{recombinant}$ human epidermal growth factor (EGF; R\&D Systems, Minneapolis, MN, USA), $10 \mathrm{ng} / \mathrm{ml}$ recombinant human fibroblast growth factor (FGF; R\&D Systems), and 1\% antibiotic-antimycotic solution (Welgene) and cultured in non-adherent plates.

Cell kinetic assay. To examine cell proliferation rates, MDA-MB-231 cells were plated at different concentrations (3,000-20,000 cells/well) into 96-well plates under nonadherent (see above), or monolayer culture conditions. After 4 days, premixed cell proliferation reagent WST-8 (Dojindo Laboratories, Kumamoto, Japan) was added to each well and the absorbance of the water-soluble formazan produced by viable cells was measured at $450 \mathrm{~nm}$ according to the manufacturer's instructions.

Cytotoxicity assay. To compare the chemo-sensitivity of cells in the TS and 2D culture systems, several chemotherapeutics including doxorubicin (Sigma, St. Louis, MO, USA), paclitaxel (Sigma), lapatinib (a dual ErbB1/ErbB2 inhibitor; LC Laboratories, Woburn, MA, USA), U0126 (a MEK inhibitor;
LC Laboratories), or LY294002 (a PI3K/AKT inhibitor; LC Laboratories) were added into cells grown in either adherent or non-adherent 96-well plates and cell viabilities were measured 3 days later. To examine the combinational effects of doxorubicin and lapatinib, cells were treated with doxorubicin in a range of $0.2-1 \mu \mathrm{M}$ in the presence of $5 \mu \mathrm{M}$ lapatinib.

Flow cytometry analysis: cell cycle analysis and doxorubicin uptake. For cell cycle analysis, cells grown in 2D or TS culture for 4 days were trypsinized after washing with PBS, centrifuged at 1,000 rpm for $3 \mathrm{~min}$ and fixed in cold $70 \%$ ethanol. After centrifugation, the cells were washed with PBS containing $2 \%$ FBS and stained in the dark with $20 \mu \mathrm{g} / \mathrm{ml}$ propidium iodide (Sigma) and $200 \mu \mathrm{g} / \mathrm{ml}$ RNase A (Sigma) for $30 \mathrm{~min}$ at room temperature. The cells were analyzed by FACS Calibur II flow cytometry (Becton Dickinson Biosciences, San Jose, CA, USA). To measure intracellular doxorubicin accumulations in cells grown in 2D or tumorsphere cultures, cells were seeded into adherent or non-adherent 6-well plates for 3 days. Cells were treated with $0.5 \mu \mathrm{M}$ doxorubicin for $30 \mathrm{~min}$, trypsinized, and washed twice with PBS containing $2 \%$ FBS. After resuspending in PBS containing 2\% FBS, the cells were analyzed by FACSCalibur II flow cytometry (Becton Dickinson Biosciences).

RNA extraction, RT-PCR and quantitative real-time PCR. Cells cultured under 2D and TS conditions were treated with $0.3 \mu \mathrm{M}$ doxorubicin, $5 \mu \mathrm{M}$ LY294002 or $10 \mu \mathrm{M}$ SB203580 (a p38 inhibitor; LC Laboratories) for 3 days, and harvested for RNA isolation. Total RNA was extracted using the easyBLUE $^{\mathrm{TM}}$ Total RNA Extraction kit (iNtRON Biotechnology Inc., Sungnam, Korea) and cDNA was synthesized with reverse transcriptase (Takara, Shiga, Japan). RT-PCR for cyclin D1, MDR-1, and GAPDH were conducted as previously described (15). Densitometric analysis was performed using Scion Image software (Scion Co., Frederick, MD, USA). The utilized primer sequences for the RT-PCR reactions were as follows: CyclinD1 (forward) 5'-AGCTCCTGTGCTGCGAAGT GGAAAC-3' and Cyclin D1 (reverse) 5'-AGTGTTCAAT GAAATCGTGCGGGG-3'; MDR-1 (forward) 5'-GCC TGGCAGCTGGAAGACAAATACACAAAATT-3' and MDR-1 (reverse) 5'-CAGACAGCAGCTGACAGTCCAA GAACAGGACT-3'; GAPDH (forward) 5'-ATCCCATCAC CATCTTCCAG-3' and GAPDH (reverse) 5'-TTCTAGACG GCAGGTCAGGT-3'. The real-time PCR reactions for multidrug resistance-associated protein-1 (MRP-1) and GAPDH were performed using QuantiMix SYBR green kit (Philekorea, Daejeon, Korea) in Eco Real-time PCR (Illumina, San Diego, CA, USA). mRNA expression level of MRP-1 was calculated after normalizing with GAPDH. The utilized primer sequences for the real-time PCR reactions were as follows: MRP-1 (forward) 5'-GCGAGTGTCTCCCTCAAACG-3' and MPR-1 (reverse) 5'-TCCTCACGGTGATGCTGTTC-3'; GAPDH (forward) 5'-CTGCTCCTCCTGTTCGACAGT-3 and GAPDH (reverse) 5'-CCGTTGACTCCGACCTTCAC-3'.

Western blotting. Cells grown in 2D or TS conditions were lysed with RIPA buffer $(50 \mathrm{mM} \mathrm{NaCl}, 1 \%$ Triton X-100, $1 \%$ sodium deoxycholate, $0.1 \%$ SDS, $50 \mathrm{mM}$ Tris- $\mathrm{HCl}, \mathrm{pH} 7.5$ and 2 mM EDTA). Phosphatase and protease inhibitor cocktails 
A

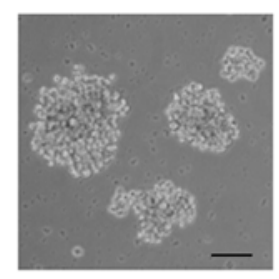

D

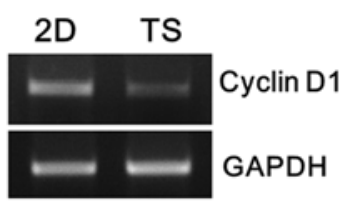

B

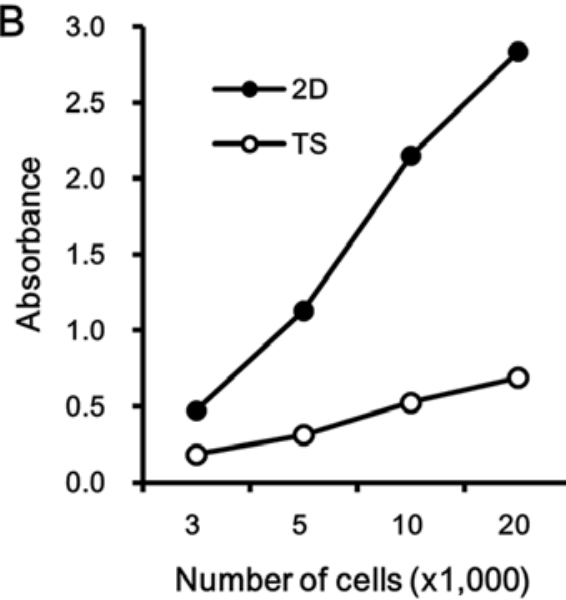

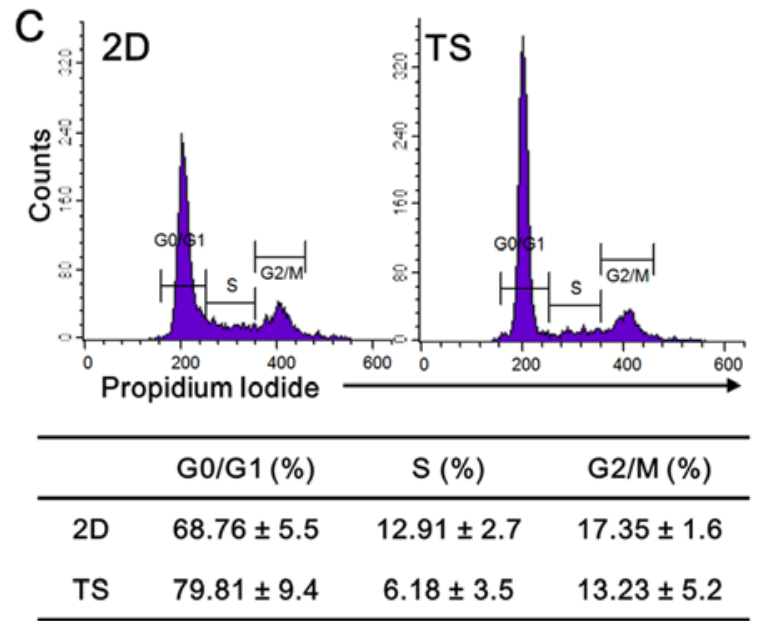

Figure 1. Sphere formation under anchorage-independent culture conditions increased the proportion of quiescent MDA-MB-231 cells. (A) Images of tumorspheres generated from MDA-MB-231 cells. Tumorspheres were cultured in non-adherent culture plates for 4 days. Scale bar, $100 \mu \mathrm{m}$. (B) Proliferation of cells grown as monolayers (2D) and tumorspheres (TS). Data indicate the absorbance of cells measured by WST-8 after incubation for 4 days. (C) Cell cycle analysis of cells grown as $2 \mathrm{D}$ or TS. Results are presented as mean $\pm \mathrm{SD}$. (D) The mRNA expression of cyclin D1 in cells grown as $2 \mathrm{D}$ and TS.

(GenDepot, Barker, TX, USA) were added immediately before use. Lysates were cleared of debris at 13,000 rpm for $10 \mathrm{~min}$, and protein concentrations were determined using bicinchoninic acid reagent (Sigma). Equal amounts of protein were separated by SDS-PAGE and transferred to polyvinylidene fluoride (PVDF) membranes, which were then blocked with 5\% non-fat skim milk in $1 \mathrm{X}$ TBS- $0.1 \%$ Tween-20 (TTBS) for $2 \mathrm{~h}$ and incubated with a primary antibody (EGFR, p-EGFR, AKT, p-AKT, ERK 1/2, p-ERK 1/2, p38, p-p38 or GAPDH; Cell Signaling, Beverly, MA, USA) overnight. HRP-conjugated secondary antirabbit antibody (Santa Cruz Biotechnology, Santa Cruz, CA, USA) diluted 1:5,000 was incubated with blots for $1 \mathrm{~h}$ at room temperature. Blots were developed using Luminescent Image Analyzer LAS-4000 (Fujifilm, Tokyo, Japan).

Statistical analysis. Statistical significance was determined using the Student's t-test. All experiments were conducted in triplicates, and results are presented as mean \pm SD. P-values of $<0.05$ were considered significant.

\section{Results}

Quiescence in tumorspheres generated from MDA-MB-231 cells. First, we cultured MDA-MB-231 breast cancer cells in non-adherent culture condition for 4 days to test their ability to form tumorspheres. As previously reported (14), tumorspheres generated from MDA-MB-231 cells exhibited much looser structures (Fig. 1A) than those derived from MCF-7 cells (data not shown). To evaluate the cell growth rates of tumorspheres and monolayer cultures, cells were plated at different concentrations (3,000-20,000 cells/well) into 96-well plates, in either non-adherent plates, or regular tissue culture plates. After 4 days of 2D or TS culture, cell viabilities were assayed by measuring WST- 8 absorbance. The overall WST- 8 readings of suspension cultures were at least three times less than those of 2D cultures, seeded with the same cell numbers (Fig. 1B). The cell cycle analysis revealed that this slow cell growth rate in tumorspheres correlated with the accumulation of cells at the $\mathrm{G} 0 / \mathrm{G} 1$ phase, showing that $79.81 \pm 9.4 \%$ cell population in tumorspheres was in the G0/G1 phase, whereas $68.76 \pm 5.5 \%$ of the 2D cultured cell population was in the G0/G1 phase (Fig. 1C). Moreover, the mRNA expression of cyclin D1, which is the first regulatory protein to drive the G1/S phase transition, was also decreased in tumorspheres (Fig. 1D). Taken together, these results confirmed that the TS culture condition enhanced the quiescent MDA-MB 231 cell population as compared with the $2 \mathrm{D}$ culture condition.

Chemoresistance of tumorspheres to doxorubicin and paclitaxel. To investigate whether culturing cells as spheres affects chemo-sensitivity, MDA-MB-231 culture under TS or 2D conditions were exposed to different concentrations of doxorubicin $(0.2-1 \mu \mathrm{M})$ or paclitaxel $(5-60 \mathrm{nM})$ for 3 days and then assessed for cell viability. It was found that tumorspheres were resistant to both doxorubicin and paclitaxel (Fig. 2A and B). In fact, the $\mathrm{IC}_{50}$ of doxorubicin for tumorspheres was at least three fold higher than its $\mathrm{IC}_{50}$ for $2 \mathrm{D}$ cultured cells (Fig. 2A). Similarly, tumorspheres exhibited significant resistance to paclitaxel with an $\mathrm{IC}_{50}$ value ten times that of $2 \mathrm{D}$ cultured cells (Fig. 2B).

We further analyzed intracellular doxorubicin accumulation using flow cytometry (16). Cells grown from either monolayer or tumorsphere cultures were treated with $0.5 \mu \mathrm{M}$ doxorubicin for $30 \mathrm{~min}$ and then doxorubicin fluorescence was analyzed by flow cytometry. The treatment of 2D cultured cells with doxorubicin for 30 min caused a right-shift of fluorescence intensity of doxorubicin as compared with untreated MDA-MB-231 cells, confirming the intracellular accumulation of doxorubicin within cells. On the other hand, the fluorescence intensity of tumorspheres was lower than that of monolayer cultured cells (Fig. 2C), implying that the decreased accumulation of intracellular doxorubicin may contribute to chemoresistance of tumorspheres.

It has been well established that the intracellular accumulation of doxorubicin is associated with the expression of the ATP-binding cassette (ABC) family of drug transporters (17), and therefore, we examined the mRNA expression levels of multidrug resistance (MDR-1) and MRP-1. It was observed 
A

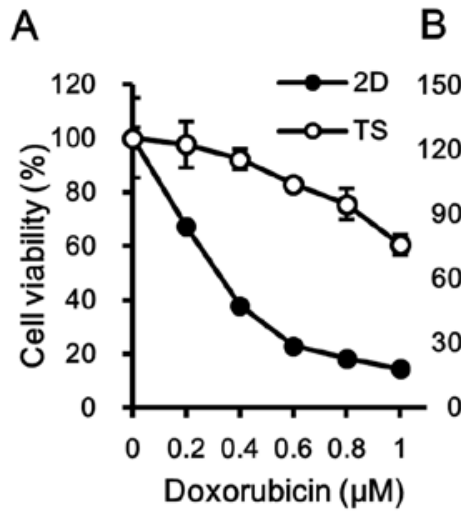

B

C

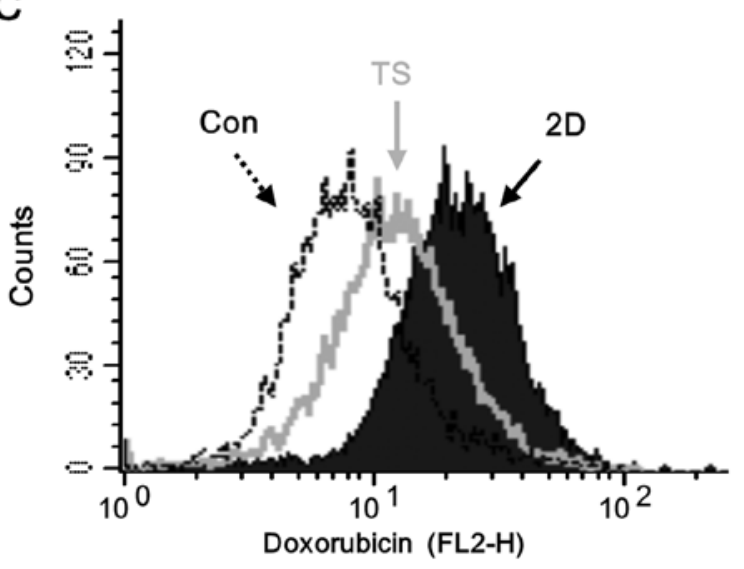

D

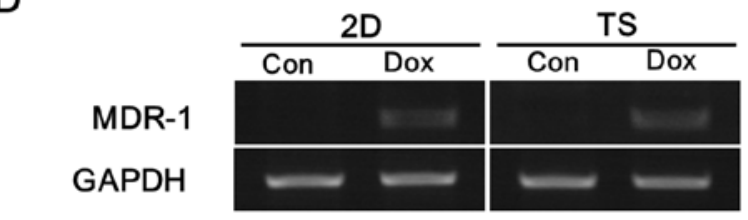

E

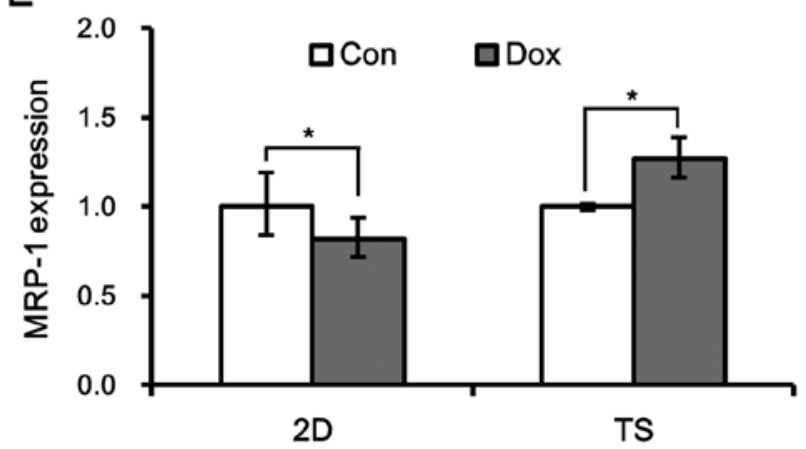

Figure 2. Tumorspheres comprised of quiescent MDA-MB-231 cells show resistance mediated by MRP-1 to chemotherapeutic agents. Monolayer (2D) or tumorsphere (TS) cultured MDA-MB-231 cells were treated with (A) doxorubicin (Dox) or (B) paclitaxel for 3 days. Cell viability was assessed using WST-8 reagent. Results are the mean of three independent experiments performed in triplicate. Error bars indicate SD of means. (C) Intracellular Dox accumulations in TS (gray lined histogram) and 2D (filled histogram) cultured cells. 2D and TS cultured cells were treated with $0.5 \mu \mathrm{M}$ Dox or vehicle control (Con) for $30 \mathrm{~min}$ and analyzed by flow cytometry. (D) Doxinduced MDR-1 expression in 2D and TS cultured cells as determined by RT-PCR. Cells were treated with $0.3 \mu \mathrm{M}$ Dox or vehicle control for 3 days. (E) Dox-induced MRP-1 expression in 2D and TS cultured cells as determined by quantitative real-time PCR. Error bars represent SD of means. ${ }^{*} \mathrm{P}<0.05$.

that the expression of MDR-1 was similarly induced by doxorubicin in TS and 2D cultured cells (Fig. 2D). However, mRNA expression of MRP-1 was induced in tumorspheres by doxorubicin but not in monolayer cultures (Fig. 2E), suggesting that MRP-1, and not MDR-1, was involved in the chemoresistance demonstrated by tumorspheres.

Enhanced EGFR signaling in tumorspheres. To elucidate the molecular mechanisms responsible for the chemoresistance of tumorspheres, we first analyzed which signaling pathways are upregulated in the tumorspheres. Western blot analysis showed that the EGFR signaling pathway was more activated in TS than in 2D cultured cells (Fig. 3A). Furthermore, the phosphorylation of EGFR in tumorspheres was associated with concomitant increases in the phosphorylations of ERK1/2 and AKT.

We next explored the role of the EGFR signaling pathway in the formation of tumorspheres. Cells were treated with lapatinib, U0126, or LY294002 to block the EGFR, MAPK, and PI3K/AKT signaling pathways, respectively, and then cell viabilities were assessed. Of note, responses to lapatinib were similar for TS and 2D cultured cells (Fig. 3B), whereas TS cultured cells were less sensitive to U0126 and to LY29400 (Fig. 3C and D). These results suggested that the EGFR signaling pathway plays an important role in mediating the survival of cells in the quiescent state rather than pathways downstream of EGFR, such as, the MAPK and PI3K/AKT pathways.

Lapatinib sensitized tumorspheres to doxorubicin by inhibiting the expression of MRP-1. We next examined whether the blockade of EGFR signaling by lapatinib enhanced cytotoxic effect of doxorubicin on quiescent MDA-MB-231 cells. Cells were treated with different concentrations of doxorubicin in the presence of $5 \mu \mathrm{M}$ lapatinib for 3 days and the cell viability was measured. Noteworthy, the chemosensitivity of tumorspheres to doxorubicin was more enhanced by lapatinib than that of 2D cultured cells (Fig. 4A and B). As shown in Fig. 4A, treatment with lapatinib mildly increased cytotoxicity of doxorubicin in the monolayer culture but the chemosensitivity of tumorspheres was dramatically increased in the presence of lapatinib (Fig. 4B). In fact, the $60 \%$ cell viability after treatment with $1 \mu \mathrm{M}$ of doxorubicin was significantly decreased to $<5 \%$ when TS cultured cells were treated with $1 \mu \mathrm{M}$ doxorubicin in the presence of $5 \mu \mathrm{M}$ lapatinib (Fig. 4B).

Since we found that increased MRP-1 expression was responsible for the chemoresistance of tumorspheres (Fig. 2E), we tested whether treatment with lapatinib affected the expression of MRP-1 in doxorubicin-treated tumorspheres. As shown in Fig. 4C, doxorubicin-induced MRP-1 expression was significantly suppressed in the presence of lapatinib, suggesting that lapatinib sensitizes tumorspheres to doxorubicin by inhibiting the expression of MRP-1.

Lapatinib inhibits doxorubicin-induced MRP-1 expression by inhibiting PI3K/AKT and p38 MAPK signaling pathways. To obtain more insight into the mechanism underlying the inhibitory effects of lapatinib against doxorubicin-induced MRP-1 expression in tumorspheres, we first analyzed the effects of lapatinib on EGFR and its downstream signaling pathways. Treatment with lapatinib was found to inhibit the phosphorylation of EGFR and that of AKT and p38 in doxorubicin-treated 
A

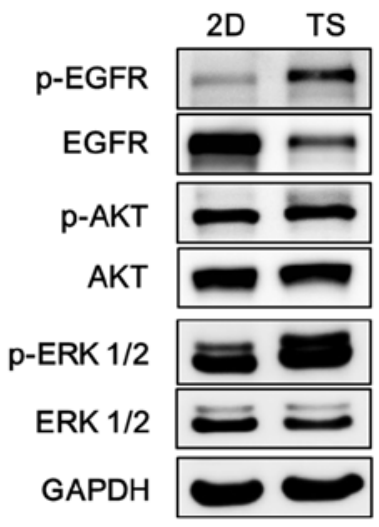

B

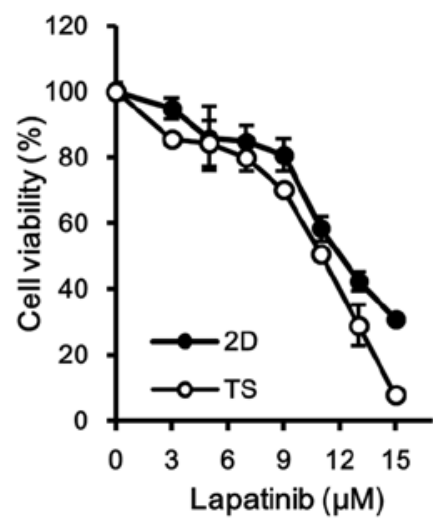

C

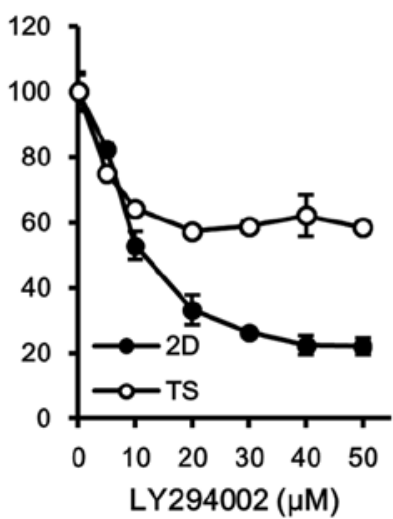

D

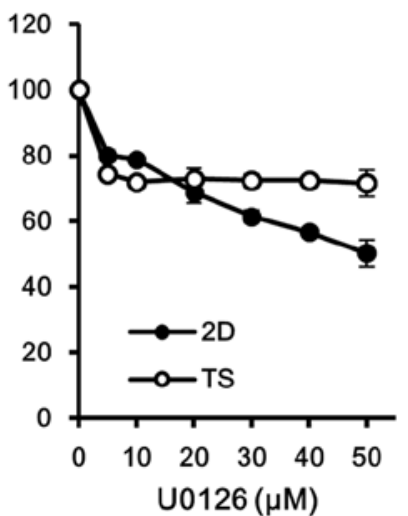

Figure 3. Enhanced epidermal growth factor receptor (EGFR) signaling in tumorspheres. (A) Whole cell lysates of tumorsphere (TS) or monolayer (2D) cultured cells were analyzed by western blotting for EGFR, AKT and ERK 1/2. Chemotherapeutic responses of 2D and TS cultured cells to (B) lapatinib (an inhibitor of ErbB1 and ErbB2). (C) LY294002 (a PI3K/AKT inhibitor), or (D) U0126 (a MEK1/2 inhibitor). After treatment with each inhibitor for 3 days, cell viability was assessed using WST-8 reagent. Results represent three independent experiments performed in triplicate. Error bars indicate the SD of means.

A

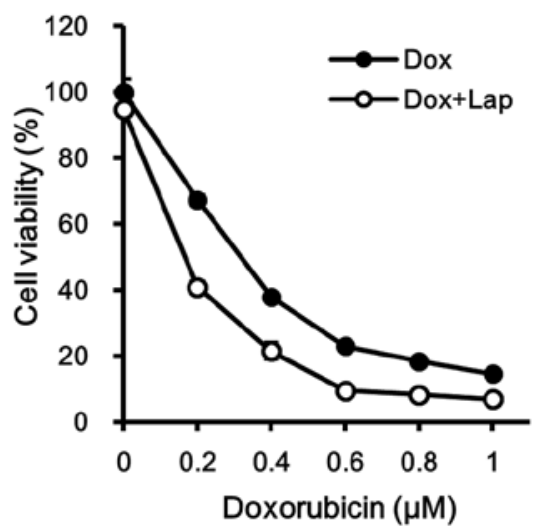

B

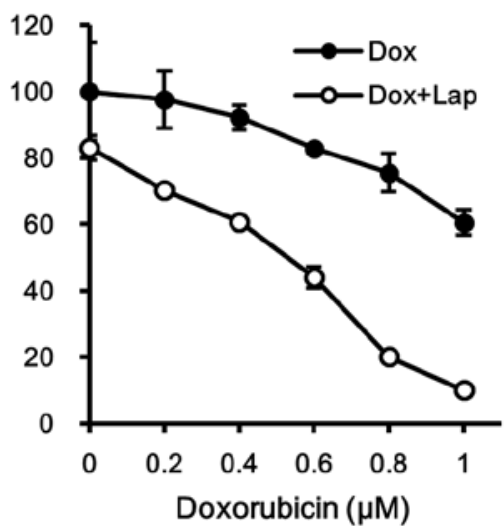

C

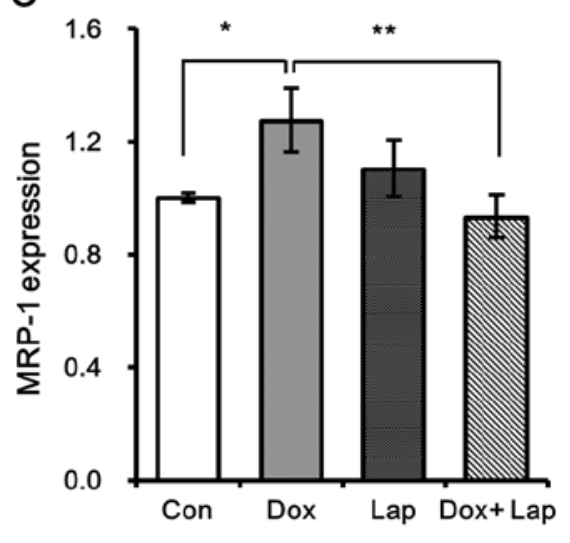

Figure 4. Lapatinib sensitizes tumorspheres to doxorubicin (Dox) by inhibiting the expression of MRP-1. Synergistic cytotoxic effects of Dox and lapatinib (Lap) on (A) monolayer (2D) cultured cells and on (B) tumorsphere (TS) cultured cells. MDA-MB-231 cells were treated with various concentrations of Dox and $5 \mu \mathrm{M}$ lapatinib (Dox+Lap) for 3 days and viabilities were assessed using WST-8 reagent. (C) Inhibitory effect of lapatinib on Dox-induced MRP-1 expression in tumorspheres. Tumorspheres were treated with $0.3 \mu \mathrm{M}$ Dox and/or $5 \mu \mathrm{M}$ Lap for 3 days and the mRNA expression of MRP-1 was analyzed by quantitative real-time PCR. Results represent three independent experiments performed in triplicate. Error bars represent $\mathrm{SD}$ of means. ${ }^{*}<0.05,{ }^{* *} \mathrm{P}<0.01$.

A

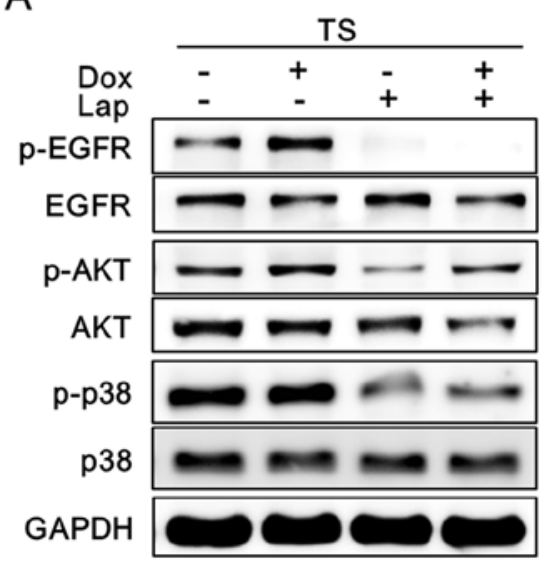

B

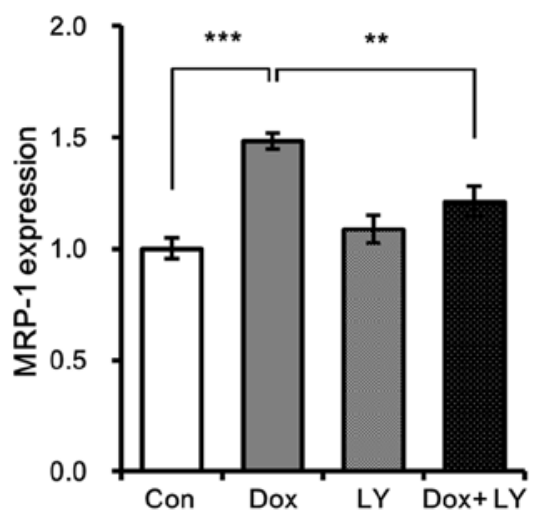

C

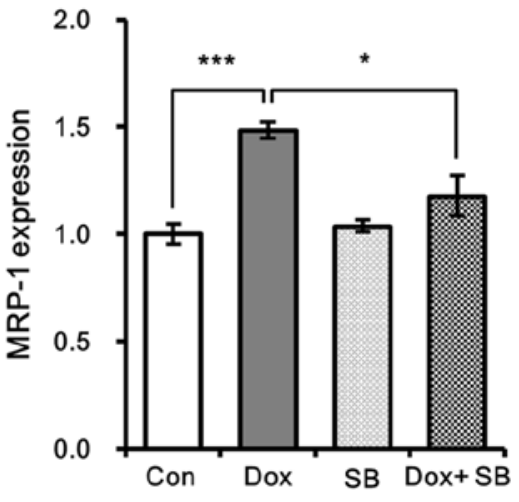

Figure 5. Lapatinib inhibits MRP-1 expression via PI3K/AKT and p38 MAPK pathways in tumorspheres. (A) Cell lysates from tumorspheres (TS) treated with $0.3 \mu \mathrm{M}$ doxorubicin (Dox) and/or $5 \mu \mathrm{M}$ lapatinib for 3 days were analyzed by western blotting for EGFR, AKT, and p38. Downregulation of Dox-induced MRP-1 expression by (B) LY294002 and by (C) SB203580 (a p38 inhibitor) in tumorspheres. TS were treated with $0.3 \mu \mathrm{M}$ Dox and/or $5 \mu \mathrm{M}$ LY294002 or $10 \mu \mathrm{M} \mathrm{SB} 203580$ for 3 days and the mRNA expression of MRP-1 was analyzed by quantitative real-time PCR. Results represent three independent experiments performed in triplicate. Error bars on the graph indicate the SD of means. ${ }^{*} \mathrm{P}<0.05,{ }^{* *} \mathrm{P}<0.01,{ }^{* * *} \mathrm{P}<0.001$. 
tumorspheres (Fig. 5A). Furthermore, blockades of the PI3K/ AKT or p38 MAPK signaling pathways with $5 \mu \mathrm{M}$ LY294002 or $10 \mu \mathrm{M}$ SB203580 remarkably decreased the expression of MRP-1 in doxorubicin-treated tumorspheres (Fig. 5B and C), indicating the involvement of PI3K/AKT and p38 MAPK signaling pathways in the expression of MRP-1 in tumorspheres. These observations suggest that lapatinib inhibits doxorubicin-induced MRP-1 expression by inhibiting EGFR signaling and its downstream PI3K/AKT and p38 MAPK signaling pathways.

\section{Discussion}

In this study, we utilized tumorsphere cultures to seek better strategies to overcome chemoresistance based on the eradication of quiescent cell population in MDA-MB-231 human breast cancer cells. Tumorsphere culture has been widely adapted to detect and propagate human breast cancer stem cells in stem cell biology $(5,6,8)$. However, several studies reported that the formation of tumorspheres does not always predict cancer stem cell enrichment and disagreed on considering it as a suitable in vitro culturing method for cancer stem cells because the formation of tumorspheres is influenced by factors such as cell density and culture duration $(14,18,19)$. Despite ongoing arguments about the enrichment of cancer stem cells in tumorspheres, the generation of tumorspheres confers interesting and unique features such as quiescence $(20,21)$. Previously, we showed that most cells in tumorsphere cultures are quiescent, whereas cells in monolayer culture have a high mitotic index (9). Similar to this study, the sphere-forming population of hepatoma cells contained a higher proportion of cells in the G0/G1 phase than the same cells cultured as monolayers (22). Since the quiescence is one of the traits in understanding the contribution of cancer stem cells to chemoresistance, we previously optimized tumorsphere cultures for in vitro screening methods for evaluating chemotherapeutics against quiescent cell population (9).

In the present study, tumorspheres generated from MDA-MB-231 cells exhibited chemoresistance to both doxorubicin and paclitaxel. However, we found that the epidermal growth factor receptor (EGFR) signaling pathway was more activated in TS than in 2D cultured cells and this enhanced activation of EGFR signaling in tumorspheres mediated survival of cells in quiescent state. EGFR is a member of the ErbB family of receptors and its activation by specific ligand binding triggers several signal transduction cascades, principally the $\mathrm{PI} 3 \mathrm{~K} / \mathrm{AKT}$ and MAPK pathways, leading to cell proliferation, adhesion, and migration $(23,24)$. In human tumors, EGFR and the other three members of the EGFR family, HER2, HER3, and HER4, are often overexpressed or dysregulated, which promotes tumor growth and/or progression $(23,24)$. EGFR is frequently overexpressed in triple-negative breast cancer and is emerging as a therapeutic target (25). Although the use of single-agent of tyrosine kinase inhibitors targeting EGFR in triple-negative breast cancer patients have produced the disappointing results $(26,27)$, several studies have reported that cytotoxic chemotherapy in combination with EGFR inhibition has shown promising results in treatment of breast cancer patients $(28,29)$. Lapatinib is an orally active small molecule, which inhibits the tyrosine kinases of EGFR and HER2, and has been approved by the FDA in combination with other anticancer agents for the treatment of HER2-positive breast cancers (30). More recent studies reported that lapatinib enhanced the cytotoxic effect of chemotherapeutics including paclitaxel, vincristine, and topotecan by inhibiting the drug efflux function of $\mathrm{ABC}$ transporters, such as P-glycoprotein (P-gp), MRPs, or ABCG2 (BCRP) transporters (31-33).

$\mathrm{ABC}$ transporters have been linked to the development of resistance to anticancer drugs as they are involved in the ATP-dependent efflux of xenobiotics or chemotherapeutics from cells and tissues $(34,35)$. Consistent with other studies, our results also show that blockade of the EGFR signaling pathway by lapatinib significantly increased the anticancer activities of doxorubicin on quiescent MDA-MB-231 cells by inhibiting the expression of MRP-1. However, the inhibition of MRP-1 expression may be insufficient to increase the anticancer activity of doxorubicin, because we observed that, unlike lapatinib, the blockade of the PI3K/AKT or p38 MAPK signaling pathways with LY294002 or SB203580, respectively, did not increase the cytotoxic effect of doxorubicin on tumorspheres although they were able to suppress the expression of MRP-1 in tumorspheres. These observations suggest that the synergistic effects of lapatinib and doxorubicin may arise from the inhibition of MRP-1 expression and the inhibition of EGFR-mediated survival signaling pathways.

In summary, we propose that although EGFR inhibition alone does not represent an effective therapeutic approach to triple-negative breast cancer, treatment with lapatinib in combination with cytotoxic chemotherapy may provide a useful approach to improve clinical responses by eradicating the quiescent cell population.

\section{Acknowledgements}

This study was supported by a grant from the National R\&D Program for Cancer Control, Ministry of Health \& Welfare, Republic of Korea (no. 1320060).

\section{References}

1. Moore N and Lyle S: Quiescent, slow-cycling stem cell populations in cancer: A review of the evidence and discussion of significance. J Oncol pii: 396076, 2011.

2. Al-Hajj M, Wicha MS, Benito-Hernandez A, Morrison SJ and Clarke MF: Prospective identification of tumorigenic breast cancer cells. Proc Natl Acad Sci USA 100: 3983-3988, 2003.

3. Tirino V, Desiderio V, Paino F, De Rosa A, Papaccio F, La Noce M, Laino L, De Francesco F and Papaccio G: Cancer stem cells in solid tumors: An overview and new approaches for their isolation and characterization. FASEB J 27: 13-24, 2013.

4. Li L and Bhatia R: Stem cell quiescence. Clin Cancer Res 17: 4936-4941, 2011.

5. Dontu G, Abdallah WM, Foley JM, Jackson KW, Clarke MF, Kawamura MJ and Wicha MS: In vitro propagation and transcriptional profiling of human mammary stem/progenitor cells. Genes Dev 17: 1253-1270, 2003.

6. Farnie G, Clarke RB, Spence K, Pinnock N, Brennan K, Anderson NG and Bundred NJ: Novel cell culture technique for primary ductal carcinoma in situ: Role of Notch and epidermal grow th factor receptor signaling pathways. J Natl Cancer Inst 99: 616-627, 2007.

7. Grimshaw MJ, Cooper L, Papazisis K, Coleman JA, Bohnenkamp HR, Chiapero-Stanke L, Taylor-Papadimitriou J and Burchell JM: Mammosphere culture of metastatic breast cancer cells enriches for tumorigenic breast cancer cells. Breast Cancer Res 10: R52, 2008. 
8. Ponti D, Costa A, Zaffaroni N, Pratesi G, Petrangolini G, Coradini D, Pilotti S, Pierotti MA and Daidone MG: Isolation and in vitro propagation of tumorigenic breast cancer cells with stem/progenitor cell properties. Cancer Res 65: 5506-5511, 2005.

9. Kim S and Alexander CM: Tumorsphere assay provides more accurate prediction of in vivo responses to chemotherapeutics. Biotechnol Lett 36: 481-488, 2014.

10. Reis-Filho JS and Tutt AN: Triple negative tumours: A critical review. Histopathology 52: 108-118, 2008.

11. Lund MJ, Trivers KF, Porter PL, Coates RJ, Leyland-Jones B, Brawley OW, Flagg EW, O'Regan RM, Gabram SG and Eley JW: Race and triple negative threats to breast cancer survival: A population-based study in Atlanta, GA. Breast Cancer Res Treat 113: 357-370, 2009.

12. Sorlie T, Tibshirani R, Parker J, Hastie T, Marron JS, Nobel A, Deng S, Johnsen H, Pesich R, Geisler S, et al: Repeated observation of breast tumor subtypes in independent gene expression data sets. Proc Natl Acad Sci USA 100: 8418-8423, 2003.

13. Isakoff SJ: Triple-negative breast cancer: Role of specific chemotherapy agents. Cancer J 16: 53-61, 2010.

14. Shaw FL, Harrison H, Spence K, Ablett MP, Simões BM, Farnie G and Clarke RB: A detailed mammosphere assay protocol for the quantification of breast stem cell activity. J Mammary Gland Biol Neoplasia 17: 111-117, 2012.

15. Kim S, Chun SY, Lee DH, Lee KS and Nam KS: Mineral-enriched deep-sea water inhibits the metastatic potential of human breast cancer cell lines. Int J Oncol 43: 1691-1700, 2013

16. Karukstis KK, Thompson EH, Whiles JA and Rosenfeld RJ: Deciphering the fluorescence signature of daunomycin and doxorubicin. Biophys Chem 73: 249-263, 1998.

17. Shen F, Chu S, Bence AK, Bailey B, Xue X, Erickson PA, Montrose MH, Beck WT and Erickson LC: Quantitation of doxorubicin uptake, efflux, and modulation of multidrug resistance (MDR) in MDR human cancer cells. J Pharmacol Exp Ther 324 95-102, 2008.

18. Calvet CY, André FM and Mir LM: The culture of cancer cell lines as tumorspheres does not systematically result in cancer stem cell enrichment. PLoS One 9: e89644, 2014.

19. Pastrana E, Silva-Vargas V and Doetsch F: Eyes wide open: A critical review of sphere-formation as an assay for stem cells. Cell Stem Cell 8: 486-498, 2011

20. Guttilla IK, Phoenix KN, Hong X, Tirnauer JS, Claffey KP and White BA: Prolonged mammosphere culture of MCF-7 cells induces an EMT and repression of the estrogen receptor by microRNAs. Breast Cancer Res Treat 132: 75-85, 2012.

21. Manuel Iglesias J, Beloqui I, Garcia-Garcia F, Leis O, VazquezMartin A, Eguiara A, Cufi S, Pavon A, Menendez JA, Dopazo J, et al: Mammosphere formation in breast carcinoma cell lines depends upon expression of E-cadherin. PLoS One 8: e77281, 2013.

22. Uchida Y, Tanaka S, Aihara A, Adikrisna R, Yoshitake K, Matsumura S, Mitsunori Y, Murakata A, Noguchi N, Irie T, et al: Analogy between sphere forming ability and stemness of human hepatoma cells. Oncol Rep 24: 1147-1151, 2010.
23. Grant S, Qiao L and Dent P: Roles of ERBB family receptor tyrosine kinases, and downstream signaling pathways, in the control of cell growth and survival. Front Biosci 7: d376-d389, 2002.

24. Normanno N, De Luca A, Bianco C, Strizzi L, Mancino M, Maiello MR, Carotenuto A, De Feo G, Caponigro F and Salomon DS: Epidermal growth factor receptor (EGFR) signaling in cancer. Gene 366: 2-16, 2006.

25. Corkery B, Crown J, Clynes M and O'Donovan N: Epidermal growth factor receptor as a potential therapeutic target in triplenegative breast cancer. Ann Oncol 20: 862-867, 2009.

26. Baselga J, Albanell J, Ruiz A, Lluch A, Gascón P, Guillém V, González S, Sauleda S, Marimón I, Tabernero JM, et al: Phase II and tumor pharmacodynamic study of gefitinib in patients with advanced breast cancer. J Clin Oncol 23: 5323-5333, 2005.

27. Dickler MN, Cobleigh MA, Miller KD, Klein PM and Winer EP: Efficacy and safety of erlotinib in patients with locally advanced or metastatic breast cancer. Breast Cancer Res Treat 115: 115-121, 2009.

28. Coley HM, Shotton CF, Ajose-Adeogun A, Modjtahedi H and Thomas H: Receptor tyrosine kinase (RTK) inhibition is effective in chemosensitising EGFR-expressing drug resistant human ovarian cancer cell lines when used in combination with cytotoxic agents. Biochem Pharmacol 72: 941-948, 2006.

29. Molina JR, Kaufmann SH, Reid JM, Rubin SD, Gálvez-Peralta M, Friedman R, Flatten KS, Koch KM, Gilmer TM, Mullin RJ, et al: Evaluation of lapatinib and topotecan combination therapy: Tissue culture, murine xenograft, and phase I clinical trial data. Clin Cancer Res 14: 7900-7908, 2008

30. Mukherjee A, Dhadda AS, Shehata M and Chan S: Lapatinib: A tyrosine kinase inhibitor with a clinical role in breast cancer. Expert Opin Pharmacother 8: 2189-2204, 2007.

31. Dai CL, Tiwari AK, Wu CP, Su XD, Wang SR, Liu DG, Ashby CR Jr, Huang Y, Robey RW, Liang YJ, et al: Lapatinib (Tykerb, GW572016) reverses multidrug resistance in cancer cells by inhibiting the activity of ATP-binding cassette subfamily B member 1 and G member 2. Cancer Res 68: 79057914,2008

32. Perry J, Ghazaly E, Kitromilidou C, McGrowder EH, Joel S and Powles T: A synergistic interaction between lapatinib and chemotherapy agents in a panel of cell lines is due to the inhibition of the efflux pump BCRP. Mol Cancer Ther 9: 3322-3329, 2010.

33. Shukla S, Chen ZS and Ambudkar SV: Tyrosine kinase inhibitors as modulators of $\mathrm{ABC}$ transporter-mediated drug resistance. Drug Resist Updat 15: 70-80, 2012.

34. Dean M: ABC transporters, drug resistance, and cancer stem cells. J Mammary Gland Biol Neoplasia 14: 3-9, 2009.

35. Higgins $\mathrm{CF}$ : $\mathrm{ABC}$ transporters: From microorganisms to man. Annu Rev Cell Biol 8: 67-113, 1992. 